\title{
Estimation of the net reproductive number of COVID-19 in Iran
}

\author{
Yousef Moradi ${ }^{1}$, Babak Eshrati*2 (10 \\ Received: 22 Mar 2020 \\ Published: 15 Apr 2020
}

\begin{abstract}
Background: Estimating the net reproductive number of COVID-19 virus can reveal the rate and severity of the disease transmission in the Iranian society to health researchers and practitioners. Also, it signifies the importance of the disease to the community. Thus, the aim of this study was to determine the net reproductive number of COVID-19 in Iran and its different provinces.

Methods: This study was conducted on the data of COVID-19 patients in Iran from February 21 to March 8, 2020. To determine the net reproductive number of COVID-19 in Iran, the serial interval and $\beta$ value were first determined using linear regression analysis. Then, the net reproductive number values were calculated for Iran and its different provinces.

Results: The results of this study showed that the net reproductive number of COVID-19 in Iran was 1.31. Using the available data up to March 15, 2020, the highest net reproductive number was in Mazandaran, Yazd, Isfahan, Qazvin, and Zanjan provinces, with values of 2.07, 1.86, 1.84, and 1.76, respectively. The net reproductive number also showed an increasing trend in Kohgiluyeh and BoyerAhmad province.

Conclusion: It seems that although the net reproductive number of the virus had a decreasing trend in the provinces, it is still high. Therefore, sanitary measures in these provinces should be pursued more seriously. On the other hand, social distancing is a topic that should be taken into consideration, that is, social gatherings and contacts should be avoided as much as possible and strategies and facilities for this action should be developed by the government and health policymakers.
\end{abstract}

Keywords: COVID 19, Net reproductive number, Iran

Conflicts of Interest: None declared

Funding: This study was financially supported by the Deputy of Research and Technology of Iran University of Medical Sciences, Tehran, Iran (Grant no. 17592).

*This work has been published under CC BY-NC-SA 1.0 license.

Copyright $₫$ Iran University of Medical Sciences

Cite this article as: Moradi Y, Eshrati B. Estimation of the net reproductive number of COVID-19 in Iran. Med J Islam Repub Iran. 2020 (15 Apr);34:34. https://doi.org/10.47176/mjiri.34.34

\section{Introduction}

Coronaviruses are a large family of viruses, and some cause disease in humans and others in animals such as bats, camels, and cats. Coronaviruses usually cause mild disease in humans and rarely evolve to infect humans and cause severe diseases such as severe acute respiratory syndrome (SARS) that appeared in 2002 and Middle East respiratory syndrome (MERS) in $2012(1,2)$.

COVID-19 is a new variant of the Coronavirus that was

Corresponding author: Dr Babak Eshrati, eshrati.b@iums.ac.ir

1. Department of Epidemiology, School of Public Health, Iran University of Medical Sciences, Tehran, Iran

2. Preventive Medicine and Public Health Research Center, Iran University of Medical Sciences, Tehran, Iran not previously known in humans. The virus was first identified in Wuhan, Hubei Province, China, where it spread widely and consistently. Since then, it has been widely distributed in China and around the world. The COVID-19 virus is closely related to the bat Coronavirus (3). There is currently a great deal of uncertainty about how COVID-19 spreads, its severity, and other factors influencing the spread and incidence of the virus, and thus much

$\uparrow$ What is "already known" in this topic:

This was the first study to investigate the net reproductive number estimation and herd immunity of COVID-19 in all provinces of Iran.

\section{$\rightarrow$ What this article adds:}

The results of this study showed that the net reproductive number of COVID-19 in Iran was 1.31, which indicated an increasing trend since February 21, 2020, reaching its highest level (1.47) on March 3, 2020. However, it started a downtrend and reached 1.31 on March 15, 2020. 
more epidemiological and clinical research is needed to find answers in this regard. The outbreak of new Coronavirus infections has raised public health concerns among people around the world, especially in developing countries. In Iran, as of March 13, 2020, a total of 11364 people have had symptoms of COVID-19, of whom 3529 recovered and 514 died. The trend of definitive and deceased cases has been increasing in Iran. According to studies and clinical findings, the incubation period of the virus is 14 to 15 days. The net reproductive number of the disease and the virus is 4.7 to 6.6 , indicating that an average person can infect 4 to 6 people. It is also estimated that the number of cases doubles every 6 to 7 days (4-6). The first laboratoryconfirmed case of COVID-19 in Iran was identified and reported on February 19, 2020 by the Pasteur Institute of Iran.

Identifying the net reproductive number of the virus in Iran can be useful in determining the epidemic peak, disease trend, and planning for social, preventive, and therapeutic interventions. The aim of this study was to determine and estimate the net reproductive number of COVID-19 virus in Iran and its different provinces.

\section{Methods}

\section{Research data}

The data for this study included information from people with COVID-19 in Iran from February 21 to March 8, 2020. Data were collected from the daily reports of the Ministry of Health and Medical Education in Iran. All patients who tested positive for COVID-19 based on laboratory results and were reported by the Ministry of Health and Medical Education $(\mathrm{MoH})$ were included in this study. Information on the daily numbers of these patients was obtained using daily reports provided by the Ministry of Health and Medical Education (MoH) (7).

\section{Model structure}

In this study, a branching model was used to estimate the net reproductive number, in which the number of secondary cases caused by patients with symptoms was estimated using equal mean negative binomial distribution (Fig. 1). Secondary cases occur only if the infected person is not separated from others after he/she has been infected. In the next step, the cumulative number of occurrences per day and its logarithm were calculated. As data on the number of infected individuals were not available, we used the cumulative number of cases and its logarithm instead of calculating the growth rate. Then, the linear relationship between the cumulative logarithm of cases in various days was estimated by simple linear regression and the estimated $B$ value was considered to be $\Delta(8-11)$.

If neither the preinfectious nor infectious periods are short, relative to the other, a branching model can best describe the natural history, and thus the equation for Rn can be as follows $(12,14)$ :

$$
R_{n}=(1+\Delta D)\left(1+\Delta D^{\prime}\right)
$$

This equation leads to the estimation of the net reproductive number of COVID-19 in Iran at the start of the epidemic. Were $D^{\prime}$ is the average preinfectious period and $D$ the average infectious period of COVID-19 in Iran and provinces. Also, in this equation, the preinfectious and infectious periods follow the exponential distribution (1517).

\section{Statistical analysis}

All analyses were done using Excel.

\section{Results}

The results of this study showed that the net reproductive number of COVID-19 in Iran was 1.31. In Iran, this number has been rising since February 21, 2020, reaching its highest level (1.47) on March 3, 2020. However, after March third, it started a downtrend and reached 1.31 on March 15. The highest and lowest values of the net reproductive number in Iran were 1.47 and 1.23, respectively (Table 1 and Fig. 2).

The results were also analyzed by different provinces. Using the data available up to March 15, it was found that the net reproductive number was increasing only in Kohgiluyeh and Boyer Ahmad province, and thus the number of future infections in this province will rise (Table 1 and Fig. 2). However, as of March 15, the net reproductive number of COVID-19 has been declining or remaining constant in other provinces of Iran (Table 1 and Fig. 3). To date (March 15), the highest net reproductive number has been in Mazandaran, Yazd, Isfahan, Qazvin, and Zanjan provinces, with values of $2.07,1.86,1.84$, and 1.76 , respectively. The lowest values of the net reproductive number on March 15 were in Kohgiluyeh and Boyer Ahmad, Ardebil, and South Khorasan provinces, which were 1.12, 1.04, 1.12 , and 1.15, respectively (Table 1 and Fig. 2). At the beginning of this epidemic on February 21 to February 24, 2020, the highest net reproductive number was in Mazandaran, Gilan, Tehran, and Qom provinces, with values of 2.14, $1.43,1.31$, and 1.29 , respectively.

Some provinces initially had very high net reproductive number, which decreased after the passage of COVID-19

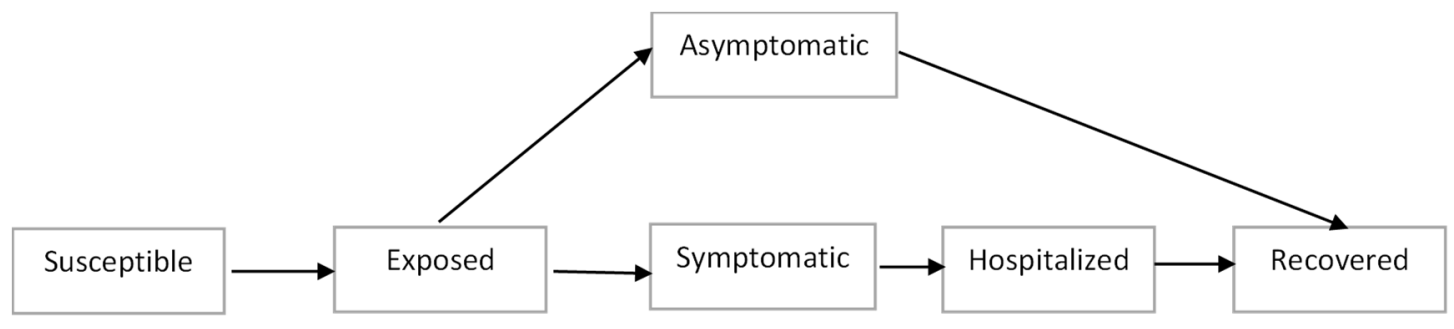

Fig. 1. Flow diagram of the compartmental model of COVID-19 in Iran 
Table 1. The net reproductive number of COVID-19 in Iran based on provinces

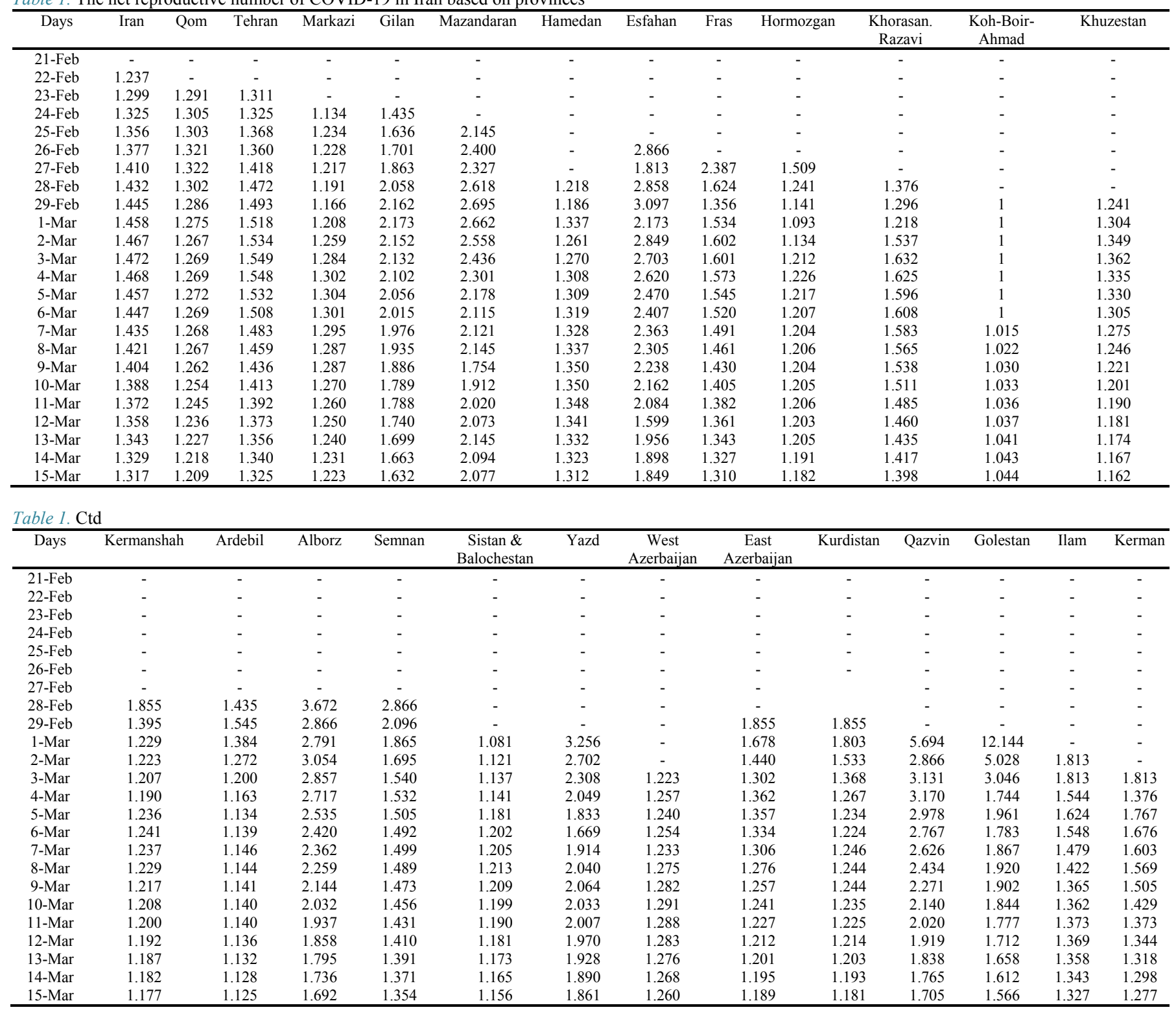

epidemic days. For example, in Golestan province, the COVID-19 net reproductive number on the first day was 12.14 , indicating that in Golestan province, every infected person could infect 12 susceptible individuals on the first day, while the net reproductive number declined to 5.02 on the second day (Table 1 and Fig. 3).

\section{Discussion}

The main aim of this study was to determine the net reproductive number of COVID-19 in Iran and its different provinces. The results showed that the net reproductive number of the virus was 1.31 in Iran. Based on the results of the present study, the net reproductive number of the disease in Iran was increasing at the beginning of the disease, reaching 1.47 on March 3, 2020, but it declined after March third and we can expect further decline in the coming days. These results prove that with continuation of interventions and cooperation of the public, the number is likely to drop below 1 in March 2020, eventually leading to a gradual decline of the epidemic. However, the changes were not the same in all provinces. For instance, in Mazandaran, Yazd, Isfahan, Qazvin, and Zanjan, the net reproductive number is still higher than other provinces, which may be due to the delay in the onset of the epidemic in these provinces, leading to a delay in their declining trend of the disease. On the other hand, people's trips to the provinces could be another reason for more contact and easier and faster spread of the virus. Determining the net reproductive number of COVID19 in Iran and other countries is of high importance, as it helps the health professionals to learn about the speed of transmission of the disease and its severity in susceptible people, which is important in providing preventive care programs in the region (18). The $\beta$ calculated in the model represents the biology of the virus and its mode of transmission. Finally, it can be argued that the net reproductive number is a combination of the number of effective contacts in a community and the rate of infection of the 


\begin{tabular}{|c|c|c|c|c|c|c|}
\hline Days & South Khorasan & North Khorasan & Zanjan & Chahar-Mahal - Bakhtiari & Busher & Lorestan \\
\hline 21-Feb & - & - & - & - & - & - \\
\hline $22-F e b$ & - & - & - & - & - & - \\
\hline $23-F e b$ & - & - & - & - & - & - \\
\hline 24-Feb & - & - & - & - & - & - \\
\hline 25 -Feb & - & - & - & - & - & - \\
\hline $26-\mathrm{Feb}$ & - & - & - & - & - & - \\
\hline $27-F e b$ & - & - & - & - & - & - \\
\hline 28 -Feb & - & - & - & - & - & - \\
\hline 29-Feb & - & - & - & - & - & 1.813 \\
\hline 1-Mar & - & - & - & - & - & 1.459 \\
\hline 2-Mar & 1.624 & - & - & - & - & 1.558 \\
\hline 3-Mar & 1.498 & - & - & - & - & 1.499 \\
\hline 4-Mar & 1.326 & - & - & - & - & 1.440 \\
\hline 5-Mar & 1.225 & - & 5.692 & - & - & 1.406 \\
\hline 6-Mar & 1.235 & 4.401 & 4.028 & 10.220 & - & 1.397 \\
\hline 7-Mar & 1.223 & 5.541 & 4.112 & 5.377 & 2.282 & 1.433 \\
\hline 8-Mar & 1.199 & 4.410 & 3.366 & 3.519 & 2.221 & 1.447 \\
\hline 9-Mar & 1.206 & 3.383 & 2.765 & 2.776 & 1.983 & 1.450 \\
\hline 10-Mar & 1.193 & 2.870 & 2.425 & 2.377 & 1.766 & 1.437 \\
\hline 11-Mar & 1.185 & 2.458 & 2.188 & 2.181 & 1.768 & 1.421 \\
\hline 12-Mar & 1.178 & 2.224 & 2.037 & 1.995 & 1.676 & 1.401 \\
\hline 13-Mar & 1.170 & 2.024 & 1.920 & 1.882 & 1.609 & 1.381 \\
\hline 14-Mar & 1.161 & 1.863 & 1.830 & 1.771 & 1.533 & 1.362 \\
\hline 15-Mar & 1.151 & 1.736 & 1.764 & 1.673 & 1.503 & 1.342 \\
\hline
\end{tabular}

COVID-19, which can be an important indicator in preventive and therapeutic measures (19).

In some provinces such as Kohgiluyeh and Boyer Ahmad, the net reproductive number is already increasing, which may be attributed to the wider presence of people outside home and participation in unnecessary gatherings and events and, finally, no adherence to personal hygiene. This province is also one of the tourist destinations of Iran, where people come to the area for holiday trips, which is also contributing to the increase in the net reproductive

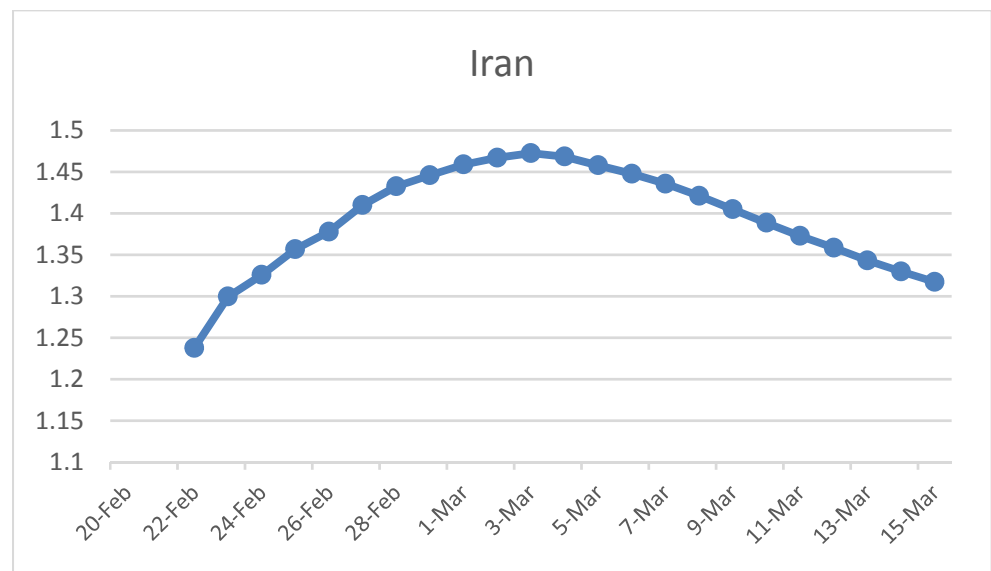

Fig. 2. The net reproductive number of COVID-19 in Iranian population

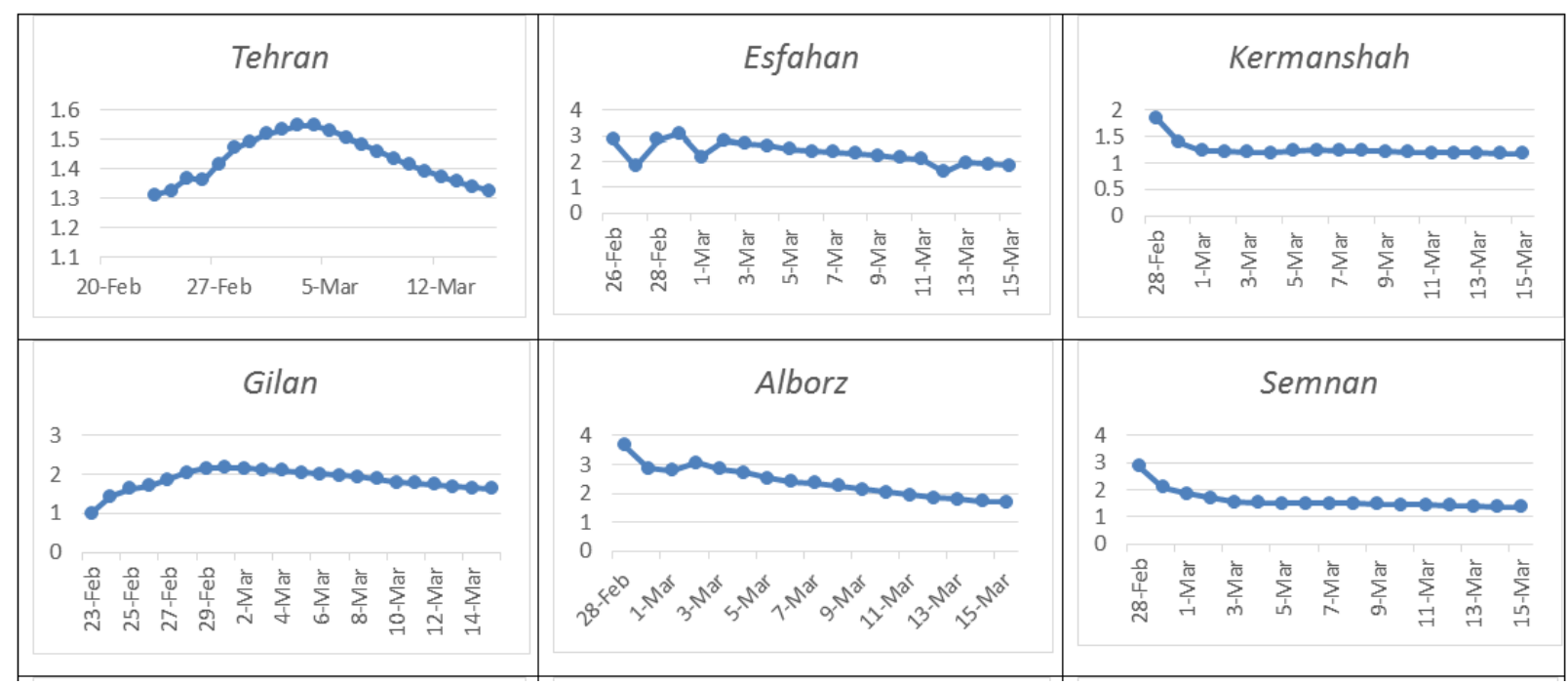

Fig. 3. The trend of net reproductive number in Iran based on provinces 


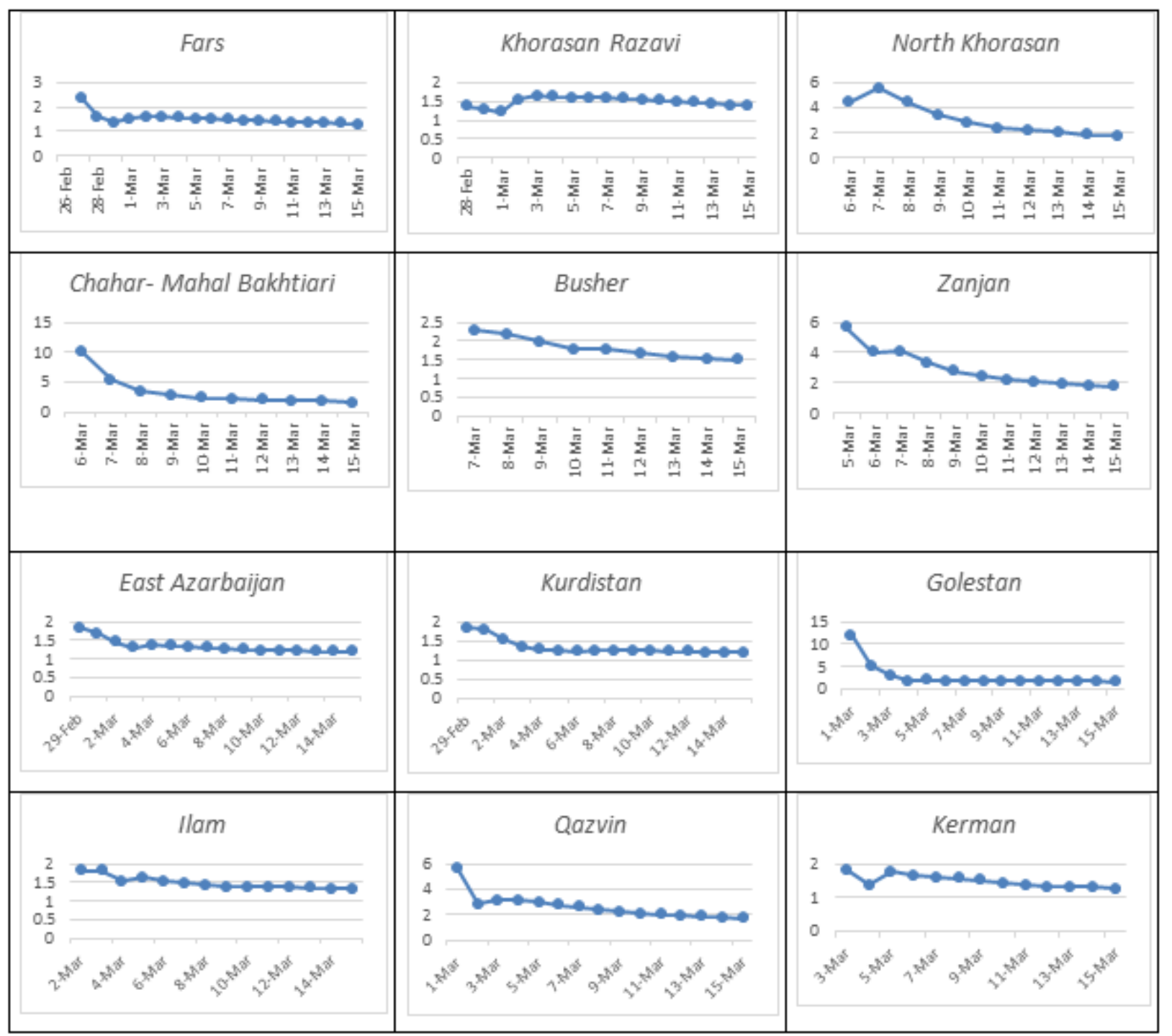

Fig. 3. Ctd

number. These estimates are based on recorded and reported data. According to studies and results published in Iran and around the world, a significant percentage of infected people have very mild symptoms and are even asymptomatic. This means that a small fraction of patients has symptoms and are diagnosed and only few will need serious medical interventions, which is not the case only in Iran. The results from China and other countries involved in this epidemic also indicate that the dangerous side effects of this infection are far less than previous forms of this virus (MERS and SARS). The obvious reason is that although this epidemic first began and had a rapid trend in Qom province in Iran, according to the latest detailed epidemiological analysis, the actual death rate has grown slowly and is approaching stability. Therefore, all statistical and mathematical models are based on probability and the available data and may be underestimated or overestimated. On the other hand, COVID-19 test is only conducted for hospitalized patients with severe symptoms and the test is not performed for many outpatient and asymptomatic patients in Iran. As a result, the majority of tests are done for high-risk individuals admitted to intensive care units. This has increased the death rate in Iran compared to some other countries, while the mortality number is for patients with serious conditions and, in this group, even if they do not have the Coronavirus, the death rate is considerable. Accordingly, death estimates should be based on national and international scientific evidence. Epidemiological models suggest that if the disease can be managed properly by public cooperation, the infection rate can decrease steadily, resulting in a substantial decrease in the number of deaths.

An important limitation of this study was its inability to estimate the sensitivity of the data provided by the surveillance system which were reported by the Iranian $\mathrm{MoH}$. However, if the reports were sustainable over time, our results would not be affected.

\section{Conclusion}

It seems that the net reproductive number of the Coronavirus in some provinces has a decreasing trend, but it is still high. Therefore, sanitary measures in these provinces should be pursued more seriously. On the other hand, the 


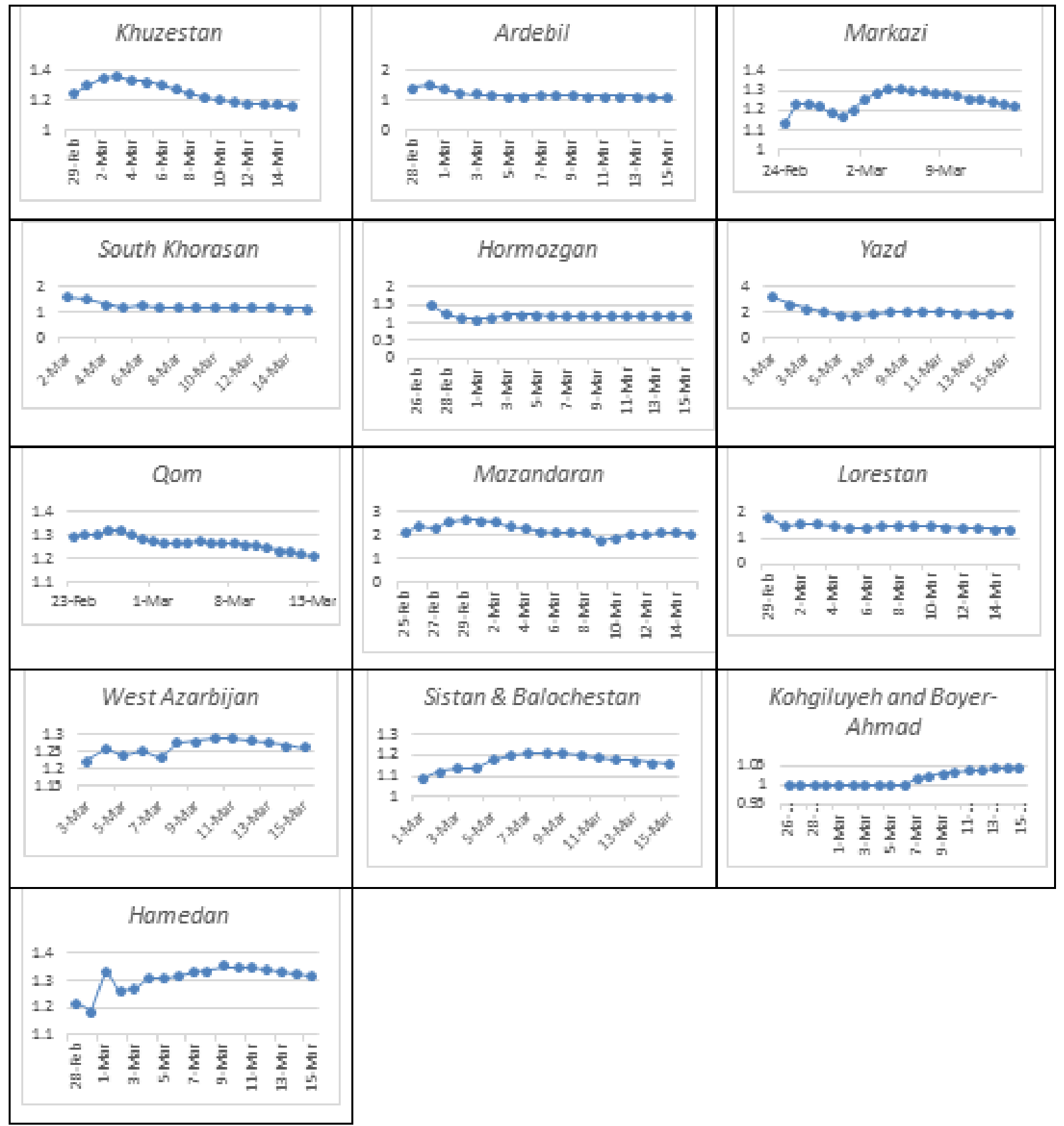

Fig. 3. Ctd

declining trend of the net reproductive number in some provinces has been increasing again, which seems to be due to poor sanitation and quarantine measures in these provinces. Suggestions to reduce the net reproductive number of the virus are as follow:

- Increasing public awareness of Coronavirus transmission by setting up information stations throughout the country; setting up 24-hour telephone counseling centers;

- Developing health centers to provide services to people and to accommodate patients with all facilities;

- Increasing cross sectoral collaboration with other organizations such as the Islamic Republic of Iran Broadcasting, etc. To provide and keep track of health issues and emphasize the importance of quarantine;
- Paying attention to social distancing such as preventing unnecessary social gatherings and unnecessary commuting.

\section{Conflict of Interests}

The authors declare that they have no competing interests.

\section{References}

1. McCall B. COVID-19 and artificial intelligence: protecting health-care workers and curbing the spread. Lancet Digit Health. 2020 Apr 1;2(4).

2. Michaels MG, La Hoz RM, Danziger Isakov L, Blumberg EA, Kumar D, Green M, et al. Coronavirus Disease 2019: Implications of Emerging Infections for Transplantation. Am J Transplant. 2020;00:15 . 
3. Cowling BJ, Leung GM. Epidemiological research priorities for public health control of the ongoing global novel coronavirus (2019-nCoV) outbreak. Eurosurveillance. 2020;25(6).

4. Wu Z, McGoogan JM. Characteristics of and important lessons from the coronavirus disease 2019 (COVID-19) outbreak in China: summary of a report of 72314 cases from the Chinese Center for Disease Control and Prevention. JAMA. 2020;323(13):1239-1242.

5. Liu Y, Gayle AA, Wilder-Smith A, Rocklöv J. The reproductive number of COVID-19 is higher compared to SARS coronavirus. J Travel Med. 2020;2(27).

6. Millán-Oñate J, Rodriguez-Morales AJ, Camacho-Moreno G, Mendoza-Ramírez H, Rodríguez-Sabogal IA, Álvarez-Moreno C. A new emerging zoonotic virus of concern: the 2019 novel Coronavirus (COVID-19). Infectio. 2020;24(3).

7.http://webda.behdasht.gov.ir/index.jsp?siteid=1\&fkeyid=\&siteid=1\&p ageid $=54782$ \&newsview $=200716$ )

8. Anderson RM, Anderson B, May RM. Infectious diseases of humans: dynamics and control: Oxford university press; 1992.

9. Kermack WO, McKendrick AG. A contribution to the mathematical theory of epidemics. Proceedings of the Royal Society of London. Series A, Containing Papers of a Mathematical and Physical Character. 1927;115(772):700-21.

10. Diekmann O, Heesterbeek JAP. Mathematical epidemiology of infectious diseases: model building, analysis and interpretation: John Wiley \& Sons; 2000.

11. Keeling MJ, Rohani P. Modeling infectious diseases in humans and animals: Princeton University Press; 2011.

12. Forsberg White L, Pagano M. A likelihood-based method for realtime estimation of the serial interval and reproductive number of an epidemic. 2008;27(16).

13. Chowell G, Nishiura H, Bettencourt LM. Comparative estimation of the reproduction number for pandemic influenza from daily case notification data. J R Soc Interface. 2007;4(12):155-66.

14. Mills CE, Robins JM, Lipsitch M. Transmissibility of 1918 pandemic influenza. Nature. 2004;432(7019):904-6.

15. Vynnycky E, Trindall A, Mangtani P. Estimates of the reproduction numbers of Spanish influenza using morbidity data. Int J Epidemiol. 2007;36(4):881-9.

16. Olson DR, Simonsen L, Edelson PJ, Morse SS. Epidemiological evidence of an early wave of the 1918 influenza pandemic in New York City. Proc Natl Acad Sci. 2005;102(31):11059-63.

17. Wallinga J, Lipsitch $M$. How generation intervals shape the relationship between growth rates and reproductive numbers. P Roy Soc B-Biol Sci. 2007;274(1609):599-604.

18. Organization WH. Preparing for the second wave: lessons from current outbreaks. Pandemic (H1N1) 2009 briefing note 9. http://www whoint/csr/disease/swineflu/notes/h1n1_second_wave20090828/en/in dexhtml.2009.

19. Stadler T, Kouyos R, von Wyl V, Yerly S, Böni J, Bürgisser P, et al. Estimating the basic reproductive number from viral sequence data. Mol Biol Evol. 2012;29(1):347-57. 